\title{
APLICABILIDADE DO SOFTWARE FAST FORWORD NA REABILITAÇÃO DOS DISTÚRBIOS DO PROCESSAMENTO AUDITIVO: RESULTADOS INICIAIS
}

\author{
Applicability of Fast ForWord software to management \\ auditory process disorders: early result
}

\author{
Sheila Andreoli Balen (1), Rosiana Massignani (2), Raquel Schillo ${ }^{(3)}$
}

\begin{abstract}
RESUMO
Tema: novas propostas de reabilitação para crianças com distúrbios do processamento auditivo associadas aos distúrbios de linguagem e de aprendizagem são necessárias para aumentar a eficácia do tratamento fonoaudiológico. Assim, o objetivo foi descrever a aplicabilidade do software Fast ForWord Language ( $F F W L$ ) na reabilitação dos distúrbios de processamento auditivo (DPA) em três crianças brasileiras. Procedimentos: estas três crianças, na faixa-etária de 9 a 14 anos, foram selecionadas pela avaliação audiológica básica, do processamento auditivo, de linguagem escrita e de consciência fonológica. Estes procedimentos foram utilizados antes e após a realização do treinamento com o software FFWL, que foi aplicado durante 80 minutos por dia, em cinco dias por semana em até oito semanas de treinamento. Foram utilizadas as seguintes estratégias: Circus sequence, Old Mac Donald's Flying Farm, Phoneme identification e Phonic Match, envolvendo detecção, discriminação, atenção sustentada e memória auditiva. Resultados: após, em média 30,67 dias de uso do FFW, observou-se adequação em duas crianças do processamento auditivo. Em uma das crianças isso não foi evidenciado mantendo-se com as mesmas alterações na reavaliação do processamento auditivo. Após a terceira semana de estimulação observou-se diminuição do interesse pelas estratégias, o que necessitou uma intervenção mais intensa e criativa dos pesquisadores. Conclusão: pode-se inferir que o FFW apresenta aplicabilidade para crianças brasileiras com distúrbio do processamento auditivo, entretanto, são necessárias novas pesquisas com uma amostra maior para verificar a eficácia deste software para crianças brasileiras.
\end{abstract}

DESCRITORES: Audição; Criança; Percepção Auditiva; Estimulação Acústica

\section{INTRODUÇÃO}

Na clínica fonoaudiológica há cada vez mais a evidência de presença de crianças com distúrbios

(1) Fonoaudióloga; Docente do Curso de Fonoaudiologia e de Especialização em Audiologia da Universidade do Vale do Itajaí; Doutora em Neurociências e Comportamento.

(2) Acadêmica do Curso de Fonoaudiologia da Universidade do Vale do Itajaí.

(3) Fonoaudióloga do Setor de Atenção a Saúde Auditiva da Universidade do Vale do Itajaí; Acadêmica do Curso de Especialização em Audiologia da Universidade do Vale do Itajaí.

Local de realização: Clínica de Fonoaudiologia e Setor de Atendimento Audiológico do Curso de Fonoaudiologia da UNIVALI/SC.

Fonte de auxílio: FINEP e UNIVALI/ProBic. do processamento auditivo em associação com os distúrbios de linguagem oral ${ }^{1}$ e/ou escrita ${ }^{2,3}$. Neste contexto, há a necessidade emergente de estudar diferentes técnicas de reabilitação auditiva que possam ser mais eficazes no tratamento destas habilidades auditivas e de linguagem, constituindo esta uma das vertentes de pesquisas na área do processamento auditivo ${ }^{4}$.

O processamento auditivo envolve mecanismos e processos do sistema auditivo responsáveis pela: lateralização e localização do som, discriminação auditiva, reconhecimento de padrões e aspectos temporais da audição, incluindo resolução, mascaramento, integração e ordenação temporais, desempenho auditivo com sinais acústicos competitivos e degradados ${ }^{4}$. O distúrbio do processamento auditivo (DPA) se refere à dificuldade no pro- 
cessamento perceptual da informação no sistema nervoso central demonstrado por um baixo desempenho em uma ou mais habilidades auditivas ${ }^{4}$.

O treinamento auditivo pode ser o recurso terapêutico adotado para minimizar habilidades alteradas e dificuldades no contexto acadêmico e social de pessoas com transtornos de aprendizagem, linguagem e/ou DPA. O termo treinamento auditivo refere-se a um conjunto de condições acústicas e/ ou tarefas que são indicadas para ativar a audição e sistemas relacionados de tal forma que sua base neural e os comportamentos auditivos associados sejam modificados gerando melhora no desempenho auditivo ${ }^{5}$. Um dos fundamentos do treinamento auditivo é a plasticidade do sistema nervoso central, definida como a mudança em células nervosas que ocorrem de acordo com as influências ambientais, sendo que se estas influências podem ser controladas e modeladas de maneira desejada, podendo ser o comportamento relacionado à plasticidade previsível. Deste modo, o treinamento pode servir como influência ambiental e as células nervosas podem representar associada mudança neural ${ }^{5}$. Portanto, na constatação do DPA um dos tratamentos indicados é o treinamento auditivo, treino este que pode ser via computador ${ }^{6}$ como vem sendo aplicado nos Estados Unidos desde a década de $90^{7}$.

Um dos programas de treinamento auditivo pioneiro e mais conhecido nos Estados Unidos, é o software Fast ForWord que foi desenvolvido pela empresa Scientific Learning ${ }^{7}$ e vem sendo estudado e aplicado há mais de 10 anos, em diferentes locais do mundo. O software Fast ForWord écomposto dos seguintes módulos Fast ForWord Language, Fast ForWord Language to Reading Prep, 1, 2, 3, 4 e 5 e Fast ForWord Middle \& High School; que tem como finalidade estimular o desenvolvimento de habilidades cognitivas que são necessárias para a eficácia da aprendizagem. $O$ foco de investigação, nesta pesquisa, foi o Fast ForWord Language, sendo que este utiliza estímulos não-verbais ou verbais com consoante-vogal. Foi o primeiro módulo a ser criado pela empresa Scientific Learning, tendo como princípio a utilização de estímulos auditivos com variação na duração, que visam desenvolver memória, atenção, processamento e seqüência relacionada à consciência fonológica, fluência, vocabulário e compreensão de fala, sendo um aspecto original deste produto seu foco nos subníveis do desenvolvimento da fala ${ }^{6,7}$.

Seus princípios são: velocidade, freqüência e intensidade do treinamento; adaptabilidade individual; desenvolvimento simultâneo de diferentes habilidades e motivação relacionada ao tempo. O produto Fast ForWord é projetado para ser uma intervenção imediata, intensiva e que propicie expe- riências relacionadas as habilidades lingüísticas. As atividades propostas pelo FFW propiciam que o cérebro realize novas conexões entre o discurso e o significado, a fim de melhorar o processamento neural $^{7}$.

A fundamentação do Fast ForWord é a manipulação dos estímulos auditivos, sendo que os estímulos verbais são expandidos no tempo com fala sintetizada e os estímulos não verbais são apresentados com graduação da diminuição da duração dos estímulos e do intervalo entre eles buscando desenvolver na criança as habilidades de resolução temporal, isto é, capacidade de detectar pequenas diferenças de duração ${ }^{6}$. Com base nestas questões, hipotetiza-se que o tipo de estímulo auditivo utilizado no software envolveria habilidades auditivas que são subliminares ou que precedem as habilidades lingüísticas e que podem também ser úteis no desenvolvimento da língua portuguesa.

Em 2005, o Curso de Fonoaudiologia da Universidade do Vale do Itajaí aprovou na Financiadora de Estudos e Projetos/Ministério de Ciências e Tecnologia o projeto: Software auxiliar na reabilitação de deficientes auditivos (SARDA), que tem como objetivo desenvolver um software para auxiliar na reabilitação de crianças com deficiência auditiva leve a moderada e com DPA baseado no Fast ForWord. Neste contexto, foram obtidos os recursos financeiros necessários para a aquisição do software Fast ForWord com uma licença, inicialmente, de seis meses. Após agosto de 2007 foram obtidos novos recursos para ampliação da licença do software para a continuidade de pesquisas com o mesmo. Desta forma, os resultados apresentados neste artigo configuram-se como resultados iniciais da aplicação do software Fast ForWord com o relato de três casos clínicos.

Salienta-se que este software é aplicado em diferentes regiões do mundo, sendo que os pesquisadores do projeto SARDA puderam presenciar esta realidade em visita a um serviço em Kuala Lumpur, na Malásia, em maio de 2006. Na América Latina a UNIVALI é a primeira Instituição a fazer uso deste software e poder verificar a aplicabilidade e, posteriormente, a eficácia do software em crianças brasileiras com distúrbios de processamento auditivo. Na literatura científica são apontados estudos controversos em relação à aplicação do Fast ForWord, principalmente, sobre os efeitos deste sobre o desenvolvimento da linguagem e da aprendizagem ${ }^{7-14}$. Porém, muitas crianças em diferentes locais do mundo têm recebido a intervenção com o Fast ForWord em clínicas, escolas públicas e privadas, evidenciando aperfeiçoamento nas habilidades auditivas ${ }^{15}$, de linguagem ${ }^{16}$ e de leitura ${ }^{17,18}$. 
Dessa forma o objetivo do presente estudo foi descrever a aplicabilidade do software Fast ForWord Language na reabilitação de três crianças com distúrbios do processamento auditivo.

\section{APRESENTAÇÃO DOS CASOS}

A amostra foi composta de três crianças brasileiras, na faixa etária de 9 a 14 anos, com diagnóstico de distúrbio do processamento auditivo (central) (DPAC).

É oportuno justificar que o número de crianças para a aplicação do software foi pequeno, em virtude do tempo de disponibilidade dos participantes - por se tratar de um treinamento auditivo diário - da disponibilidade em comum dos horários dos pesquisadores, dos participantes e também pelo tempo da licença do software Fast ForWord na UNIVALI, que foi por um período de seis meses - de julho a dezembro de 2006 - em um único computador. Assim, durante este período foi utilizado o mês de agosto para a aplicação dos procedimentos de seleção das crianças, bem como os procedimentos de referência (anteriores a estimulação com o software). Além disso, realizou-se neste mesmo mês um estudo do software Fast ForWord, a fim de conhecer os princípios, etapas e procedimentos para o uso do mesmo, bem como foi realizada experiência prática de todas as atividades terapêuticas do software passando pela experiência de uso do programa. Os meses seguintes - de setembro a dezembro - foram utilizados para aplicação do software com as crianças brasileiras.

Por meio de pesquisa documental e contato via telefone foram selecionadas sete crianças na Clínica de Fonoaudiologia da UNIVALI com histórico de queixas de dificuldades relacionadas ao processamento auditivo e/ou leitura e escrita que compareceram para avaliação audiológica e do processamento auditivo em data e horário previamente agendado. Durante a avaliação audiológica e do processamento auditivo os pais foram consultados e convidados a participar do presente estudo, nesse mesmo instante houve o esclarecimento dos riscos e benefícios da participação desse estudo. Os pais que aceitaram assinaram o Termo de Consentimento Livre e Esclarecido.

Todas as crianças foram submetidas aos seguintes procedimentos de seleção:

- Avaliação audiológica básica constando de audiometria tonal liminar, limiar de reconhecimento e recepção da fala e imitância acústica. Estes tiveram como objetivo verificar a integridade da via auditiva periférica. Esta avaliação inicial ainda forneceu dados sobre as condições de detecção do som através da audiometria tonal e dados sobre a mobilidade do sistema tímpano-ossicular através das medidas de imitância acústica.

- Avaliação de consciência fonológica: utilizando o protocolo CONFIAS. Este protocolo está subdividido em tarefas de consciência silábica e fonêmica. O intuito de aplicação deste protocolo era verificar a presença de dificuldades nesta área para delimitação do diagnóstico.

- Avaliação de leitura e de escrita: foi realizado por meio da escrita espontânea e ditado de frases, sendo observada a presença de: 1) erros fonológicos; 2) erros por transcrição de fala; e 3) erros ortográficos. A leitura foi observada de forma informal quanto à leitura silenciosa e em voz alta, bem como interpretação de um texto dado pelo pesquisador. Também foram analisadas as queixas atuais relatadas pelos pais, de dificuldades escolares, como: troca de letras na escrita, dificuldade em elaborar textos, dificuldade na leitura e em compreender o texto lido.

- Avaliação de vocabulário e fonologia: foi aplicado o teste de linguagem infantil ABFW no intuito de verificar qual o nível de desenvolvimento do vocabulário e da fonologia, auxiliando na definição do diagnóstico fonoaudiológico.

- Avaliação comportamental do processamento auditivo incluindo os seguintes testes:

- Teste de fala filtrada: o objetivo do teste foi avaliar o fechamento auditivo, utilizando monossílabos com filtragem passa-baixo apresentados de forma monótica a 50dB NS. Os sujeitos precisaram repetir uma lista de 25 monossílabos na orelha direita e depois na orelha esquerda. Observou-se o percentual de acertos de cada orelha, tendo como referência de normalidade diferença igual ou menor do que $20 \%$ do que o índice percentual de reconhecimento de fala (IPRF).

- Teste de dissílabos alternados (Staggered Spondaic Word - SSW): O teste é composto por quatro itens de treinamento e 40 itens válidos, onde 20 serão iniciados na orelha esquerda e 20 serão iniciados na orelha direita. O teste contém desta forma, 40 itens, sendo que cada item é formada por quatro palavras, cada palavra é apresentada a cada orelha, havendo uma superposição parcial entre elas. Logo, o sujeito ouviu os itens e precisou repetir as palavras na ordem ouvida. As palavras foram apresentadas a $50 \mathrm{~dB}$ NS. $O$ registro foi realizado na folha de resposta do próprio teste, anotando-se os erros e as inversões.

- Teste de dicótico de dígitos: o teste é dividido em tarefa de integração binaural - ava- 
liar a habilidade para agrupar componentes do sinal acústico em figura-fundo e identificá-los (denominá-los em termos verbais) e na tarefa de separação binaural - avaliar a escuta direcionada para cada orelha separadamente. O teste foi aplicado a $50 \mathrm{~dB}$ NS. $\mathrm{Na}$ análise do teste foi computado o número de acertos cometidos na orelha direita e na orelha esquerda nas três etapas de apresentação dos estímulos. Estabelecendo o percentual de acertos de cada orelha sendo este ainda comparado ao valor de normalidade por faixa etária: 7 a 8 anos e 11 meses = $75 \%$; 9 a 10 anos e 11 meses = 85\%; a partir dos 11 anos $=90 \%$.

- Teste de padrões de freqüência (Pitch pattern sequence test - PPS): Este procedimento constou da apresentação de três tons que diferiam entre si em duas freqüências: $1430 \mathrm{~Hz}$ (alto) e $880 \mathrm{~Hz}$ (baixo) (AUDITEC, 1997). Cada tom tinha duração de $500 \mathrm{~ms}$, com subida e queda de $10 \mathrm{~ms}$. O intervalo entre os três tons era de $300 \mathrm{~ms}$ e o intervalo entre cada seqüência de tons era de 10s. Os tons foram combinados em seis diferentes padrões de freqüência: alto-alto-baixo, alto-baixo-baixo, alto-baixo-alto, baixo-altoalto, baixo-alto-baixo e baixo-baixo-alto. Os padrões foram apresentados monoauralmente a $50 \mathrm{~dB}$ NS. Utilizou-se como parâmetro de normalidade acertos de $90 \%$ para crianças acima de nove anos.

- Teste de padrões de duração (Duration pattern sequence test - DPS): Este procedimento constou da apresentação de três tons consecutivos, que se diferenciavam entre si em duas durações diferentes: 500 ms (longo) e $250 \mathrm{~ms}$ (curto). Os tons tinham freqüência de $1000 \mathrm{~Hz}$, subida e queda de $10 \mathrm{~ms}$. Os intervalos entre os tons eram de $300 \mathrm{~ms} e$ os intervalos entre as seqüências de $7 \mathrm{~s}$. As seqüências de duração foram apresentadas monoauralmente a $50 \mathrm{~dB} N{ }^{21}$. O parâmetro de normalidade utilizado foi de $67 \%$, porém destaca-se que não existe parâmetro para crianças. Assim, destaca-se que o intuito deste teste foi observar por meio dele evoluções antes e após o treinamento, visto que este teste sofre influências do processo de aprendizagem e que, portanto, seria mais indicado em monitoramento terapêutico ${ }^{22}$.

- Teste de detecção de intervalos de silêncio (Random Gap Detection test - RGDT e Random Gap Detection Test Expandid RGDT-Exp). O teste consiste na apresentação aleatória de um tom em pares com breves intervalos entre eles. A criança foi instruída a verbalizar se ouvia um ou dois tons a cada apresentação. Consistiu de dois subtestes: $1^{\circ}$ Subteste 1: é o teste de treinamento que consistiu na apresentação de nove pares de tons de $500 \mathrm{~Hz}$, apresentados com intervalos entre os tons de forma ascendente, isto é, os mesmos vão aumentando até a criança reconheça dois e não um tom. Neste momento, iniciou-se com o RGDT com intervalos de 0 a $40 \mathrm{~ms}$, caso a criança não detectasse os intervalos nesta variação era aplicado o RGDT-expandido com intervalos de 50 a 300 ms. $2^{\circ}$ Subteste 2: consistiu na apresentação de nove pares de tons de 500, 1000, 2000 e $4000 \mathrm{~Hz}$ com aumento no intervalo entre os tons. Os estímulos foram apresentados binauralmente em intensidade confortável. O teste durou em média 10 minutos de aplicação. Os resultados encontrados foram comparados ao limiar de detecção do gap de 6.0 a $8 \mathrm{~ms}$ com desvio padrão de 2,5 a 5,3 ms.

A avaliação comportamental do processamento auditivo foi realizada tanto para definir a presença de Distúrbio do Processamento Auditivo (Central) (DPAC), quanto para verificar se havia mudanças no processamento auditivo após o uso do software FFW. Foi considerada a presença de $\mathrm{DPA}(\mathrm{C})$ quando havia pelo menos dois testes rebaixados ${ }^{4}$. Todos os procedimentos de avaliação comportamental do processamento auditivo (central) expostos anteriormente foram reaplicados ao final da estimulação com o software e, conseqüentemente, foram comparados os desempenhos de cada sujeito em cada medição no intuito de verificar se houveram mudanças nos mesmos.

Após estas avaliações, as famílias de duas crianças não consentiram em trazer seus filhos todos os dias para a estimulação com o software Fast ForWord, uma das crianças apresentou resultados normais na avaliação do processamento auditivo e outra criança desistiu ao final da estimulação com o software Fast ForWord. Logo, a amostra foi constituída por três sujeitos.

Assim, em decorrência das alterações nos processos de discriminação, atenção e memorização auditiva, bem como de transtornos de leitura e escrita, os três sujeitos foram encaminhados para a aplicação do software Fast ForWord.

A seguir serão descritas as características de cada sujeito:

O sujeito 1 (S1) do sexo feminino, com nove anos de idade na época da intervenção que durou na totalidade de 34 dias. S1 apresentava na préestimulação distúrbio do processamento auditivo caracterizado por déficit de associação auditivo- 
lingüístico ${ }^{19}$, associado a transtornos de leitura e escrita. Era calma, alegre, concentrada, participativa, sempre questionava o que estava fazendo e gostava de contar sobre suas atividades familiares e escolares. Além de mostrar-se muito interessada na aplicação do software, adorou montar um painel de pontuação e acompanhar sua própria evolução. S1 comparecia acompanhado pelo seu pai, que se mostrava interessado e acompanhava a filha todos os dias, questionando os pesquisadores sempre que julgava necessário.

O sujeito 2 (S2) do sexo masculino, apresentava 12 anos na época da intervenção que durou 32 dias. S2 apresentava na pré-estimulação distúrbio do processamento auditivo caracterizado por déficit de decodificação e de associação auditivo-lingüístico ${ }^{19}$, associado a transtornos de leitura e escrita. Durante o processo de avaliação e as intervenções diárias com o software mostrou-se, na maior parte das vezes, participativo, concentrado, interessado e realizando vários questionamentos sobre a função da intervenção com o software. Em alguns momentos mostrou-se cansado, sem vontade de realizar as atividades ou mesmo agitado. S2 vinha a Clínica de Fonoaudiologia da UNIVALI, na maior parte das vezes sozinho, chegando sempre cerca de 40 a 50 minutos antes do seu horário de intervenção. O protocolo utilizado com S2 era de 100 minutos diários, porém o mesmo apresentou algumas faltas ao longo do processo. Estas faltas na sua totalidade foram às sextas-feiras. Ao longo dos 100 minutos era realizado intervalo após duas atividades do software. Neste momento, S2 gostava de propor brincadeiras, por exemplo, de memória ("Hoje fui à feira e comprei um (a)...) ou mesmo levantar-se e ir até o toalete.

A mãe de S2, quando o acompanhava, era participativa e mostrava-se interessada na participação de seu filho na pesquisa, sempre questionava e tinha curiosidade sobre o funcionamento do estudo. Então, ela foi convidada a assistir uma aplicação do software Fast ForWord. Após assistir relatou que se surpreendeu e julgou o software interessante para o desenvolvimento de seu filho.

O sujeito 3 (S3) do sexo masculino, com 14 anos na época da intervenção que durou 26 dias, tendo sido finalizadas, em virtude das Festas Natalinas. S3 referia interesse nas atividades do software, porém apresentava sonolência e, por vezes, dificuldade e lentidão nas respostas no inicio da intervenção. Ao final da estimulação demonstrava mais facilidade $e$ rapidez. No momento das avaliações fonoaudiológicas houve a suspeita de que S3 pudesse apresentar algum comprometimento cognitivo ou neurológico associado as suas dificuldades de processamento auditivo (central) e de aprendizagem. Estas hipóte- ses não puderam ser esclarecidas, embora tenha se realizado os encaminhamentos necessários. $O$ tipo de distúrbio do processamento auditivo apresentado por S3 era, predominantemente, de integração auditiva e/ou prosódia ${ }^{19}$.

O Fast ForWord é um programa que consiste no treinamento por computador intensivo. Este foi aplicado 80 minutos por dia, cinco dias por semana ${ }^{7}$, com uma média de 30,67 dias de estimulação (mínimo de 26 dias e máximo de 34 dias de estimulação). Para a aplicação do software, inicialmente foi realizado o cadastro dos participantes, sendo criado um grupo DPA(C), registrando dados de identificação dos participantes. Na aplicação foi utilizado o módulo Fast ForWord Language Basic e Fast ForWord Language, sendo que o primeiro módulo foi utilizado apenas nos dias iniciais da estimulação com o objetivo de verificar a familiaridade dos participantes do estudo com o computador, em manipular o mouse e com a própria atividade, bem como para um treinamento pré-estimulação para adaptação com os objetivos das atividades e também para apresentar os estímulos auditivos modificados, visto que é um módulo composto com três estratégias destinadas a crianças menores de sete anos de idade.

Em seguida foi aplicado o Fast ForWord Language que consistiu de quatro estratégias terapêuticas que foram realizadas com a criança em todos os momentos ${ }^{7}$ :

- Circus sequence: nesta estratégia a criança ouvia uma seqüência de sons não-verbais com variações de freqüência e de duração e tinha que reproduzi-los clicando em dois botões. $O$ intervalo de tempo entre os sons ia diminuindo no decorrer da estratégia e conforme a criança acertava os mesmos, tornando-se assim mais rápido. Esta estratégia visou desenvolver as habilidades auditivas de detecção, discriminação e memória auditiva.

- Old Mac Donald's Flying Farm: a criança ouvia um estímulo alvo e tinha que largar o mouse quando identificasse a presença de um som diferente. Nesta estratégia o estímulo utilizado era verbal com consoante-vogal (ba, da, aba, ada) expandidos no tempo. Desta forma, nos níveis mais fáceis da estratégia o som era expandido (isto é, aumentado como fala sintetizada) e conforme a criança ia passando de nível havia diminuição da duração acústica dos fonemas até alcançar níveis normais. Esta estratégia visou desenvolver as habilidades de detecção, atenção sustentada, concentração e discriminação auditiva.

- Phoneme identification: nesta estratégia a criança ouvia um estímulo inicial verbal (conso- 
ante-vogal) e tinha que reconhecer entre dois outros estímulos qual é igual ao estímulo auditivo inicial. Assim, esta estratégia também enfocou a habilidade auditiva de discriminação auditiva, mas também necessitava das habilidades de atenção e memória auditiva.

- Phonic Match (PM): consistiu em a criança ouvir sons verbais consoante-vogal com variações de duração (expandidos na fase mais fácil do programa) e encontrar o par igual ao ouvido. É um jogo de memória auditiva. À medida que a criança avançava na estratégia o estímulo auditivo tornava-se com duração da fala normal. Esta estratégia trabalhou a habilidade auditiva de discriminação e atenção, com enfoque principal na memória auditiva de curta duração.

Este módulo - Fast ForWord Language - ainda apresenta as estratégias Phonic Words e Block Commanderque não foram utilizadas com as crianças deste estudo, pois exigem escuta de palavras e sentenças em inglês e, portanto, seria necessário o conhecimento prévio da língua inglesa.

O software não libera todas as estratégias todos os dias, há uma seqüência pré-definida pela empresa determinando qual atividade a ser liberada em cada dia de estimulação. Como os pesquisadores não utilizaram duas estratégias conforme exposto no parágrafo anterior, realizou-se a complementação da estimulação utilizando a versão demo do software. Então, eram aplicadas as atividades liberadas e, em seguida, as atividades não liberadas naquele dia na versão demo do próprio software Fast ForWord. Este procedimento não acontecia diariamente e era apenas no intuito de completar a estimulação auditiva de 80 minutos em todos os dias. É importante relatar que a versão de demonstração não registra o desempenho da criança na base do banco de dados.

Um ponto interessante do software é que ele possibilita o registro diário, podendo então ser visto o desempenho diário da criança durante a estimulação. Outro fato que pode ser usado para o percurso do participante no treinamento é a pontuação das atividades, pontuação esta que foi registrada diariamente para monitorar as evoluções observadas ao longo da aplicação do FFW no decorrer das oito semanas.

Durante a intervenção do software Fast ForWord foi preciso criar estratégias para motivar os sujeitos, por isso realizou-se uma atividade complementar e interativa, servindo então como incentivo para que as crianças realizassem as atividades com concentração, empenho e vontade. A primeira estratégia foi à criação de um Painel de Pontuação Criativo, ilustrativo, colorido e com figuras. Inicialmente anotávamos a pontuação de todas as atividades do par- ticipante, por meio de uma tabela, no dia seguinte anotávamos novamente, caso o participante ultrapassa-se a pontuação de no mínimo três das quatro atividades do dia anterior.

Além do painel de pontuação foi elaborado o Prêmio de Presença Diária que consistia como o próprio nome diz, em um prêmio pela presença diária, este era entregue aos participantes todas as sextas-feiras, se os mesmo não faltassem durante a semana. Caso faltassem, simplesmente não recebiam seu prêmio, nosso intuito era incentivar a vinda diária dos participantes na aplicação do software Fast ForWord, como forma de "recompensa" pela participação do nosso estudo, e ainda este Prêmio servia como forma de decoração para a sala de treinamento. Este prêmio consistia em adesivos e desenhos.

Após 30 dias de intervenção com o software realizou-se a segunda etapa de bateria de testes de avaliação do processamento auditivo (central), com o objetivo de analisar a aplicabilidade do software Fast ForWord em crianças brasileiras com Distúrbio do Processamento Auditivo. Desta forma, foram comparados todos os resultados obtidos antes e após a aplicação do mesmo.

Para finalizar realizou-se devolutiva aos pais dos participantes, explicando o que havia acontecido até aqui, e o que havia mudado na reavaliação, ou seja, se seu filho havia melhorado e em quais habilidades após a estimulação com o software Fast ForWord. Os mesmos receberam um relatório com os resultados das avaliações pré e pós-estimulação. Nos casos em que foi necessário a continuidade da intervenção fonoaudiológica os mesmos foram encaminhados a Clínica de Fonoaudiologia da Univali.

No intuito de facilitar a leitura dos resultados da pesquisa optou-se por apresentar a avaliação de leitura, escrita e de consciência fonológica e descrição do processo terapêutico de cada sujeito individualmente, bem como aspectos gerais de seus comportamentos para, posteriormente, apresentar os resultados da avaliação comportamental do processamento auditivo antes e após a intervenção com o software Fast ForWord Basic e Language.

Foi realizada a tabulação dos dados pré e pósestimulação de todos os participantes e realizada a análise das diferenças inter e intra-sujeitos utilizando a estatística descritiva. Por se tratar de um estudo inicial e de relato de caso optou-se por descrever cada sujeito em separado no que se refere às evoluções ou não demonstradas durante o processo de reabilitação utilizando o software e, posteriormente, comparou-se os resultados quantitativos obtidos nos procedimentos de avaliação do proces- 
samento auditivo antes e após o uso do software fast forword.

Este projeto foi aprovado pelo Comitê de Ética em Pesquisa da Universidade do Vale do Itajaí, sob o número 252/06.

\section{RESULTADOS}

A amostra desta pesquisa foi composta por três sujeitos com diagnóstico de distúrbio do processamento auditivo (central), sendo que todos tinham diagnóstico de distúrbios de leitura e escrita.

\section{Descrição do desempenho dos sujeitos no software Fast ForWord, avaliação da leitura e escrita e consciência fonológica antes e após a aplicação do software Fast ForWord.}

\section{Sujeito 1 (S1)}

As atividades realizadas por $\mathrm{S} 1$ foram: circus sequence, phonic match, phoneme identification e Old Mac Donald's Flying Farm. A mesma apresentava dificuldade em uma das atividades, a Phoneme Identification, mas precisamente nos fonemas /bi/ e /di/. Apesar disto S2 adorava esta atividade, talvez por ser um desafio para ela.
Na avaliação de escrita de S1 na pré-estimulação foram observados os seguintes aspectos: não compreensão da variação entre o modo de falar e o modo de escrever, apresentando erros fonológicos (predominância de trocas de grafemas surdos por sonoros e vice-versa e ortográficos na escrita; não realização de auto-correção e re-escritura do texto, dificuldade para organizar o texto, usou inadequadamente a separação de sílabas não utilizou pontuação e a grafia da letra estava em tamanho irregular. Em relação à leitura $\mathrm{S} 1$ apresentou alterações na fluência, não respeitando a pontuação, com dificuldade para reconhecer e discriminar as letras e apresentando também alterações na compreensão da leitura.

$\mathrm{Na}$ avaliação da leitura e escrita pós-estimulação não foi observada nenhuma modificação.

No que se refere a consciência fonológica na préestimulação S1 apresentou desempenho de $75 \%$ de acertos no nível da sílaba e $86,6 \%$ do fonema na pré-estimulação. Na pós-estimulação o desempenho no nível silábico aumentou para $97,5 \%$ e o nível fonêmico manteve-se $86,6 \%$ de acertos.

Observa-se na Figura 1 o desempenho de $\mathrm{S} 1$ ao longo dos dias de intervenção.

\section{Fast ForWord Language Completion History}

100-Minute Day 1 Protocol: Participation Day 34

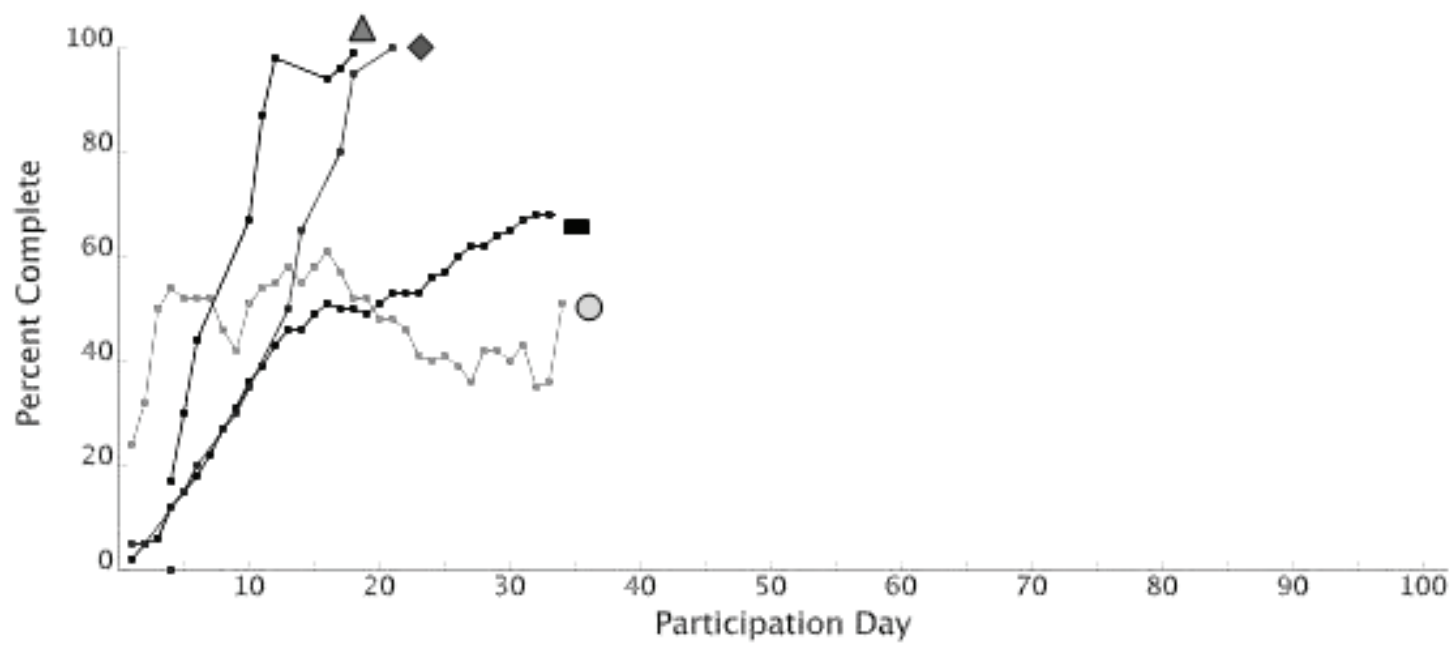

Circus Sequence $(68 \%)$

Phoneme Identification (61\%) old MacDonald's Flying Farm (99\%)

$\triangle$ Phonic Match $(100 \%)$

Fonte: Progress Tracker, Scientific Learning, 2006.

Figura 1 - Desempenho ao longo da aplicação do software FFW do sujeito 1 


\section{Sujeito 2 (S2)}

As atividades realizadas por S2 foram: circus sequence, phonic match, phoneme identification e Old Mac Donald's Flying Farm. De todas as que $\mathrm{S} 1$ demonstrava mais facilidade era a do Circus Sequence, sendo também a sua atividade preferida.

$\mathrm{Na}$ avaliação da escrita pré-estimulação observou-se erros fonológicos (predominância de trocas de grafemas surdos por sonoros e vice-versa e ortográficos, alterações na pontuação e dificuldades em organizar o texto. No que se refere a leitura não foram evidenciadas nenhuma dificuldade, apresentando adequada fluência e compreensão da leitura.
$\mathrm{Na}$ avaliação da escrita e leitura pós-estimulação constatou-se diminuição no número de erros fonológicos e ortográficos, melhora na organização espacial do texto escrito e uso adequado da pontuação com maior freqüência.

Em relação à consciência fonológica na préestimulação S2 apresentou $77,5 \%$ de acertos em tarefas de nível silábico e $80 \%$ de nível fonêmico. Na avaliação pós-estimulação observou-se $100 \%$ de acertos tanto no nível silábico quanto fonêmico, evidenciando melhora na consciência fonológica.

Observa-se na Figura 2 o desempenho de S2 ao longo dos dias de intervenção.

Fast ForWord Language Completion History

100-Minute Day 1 Protocol: Participation Day 32

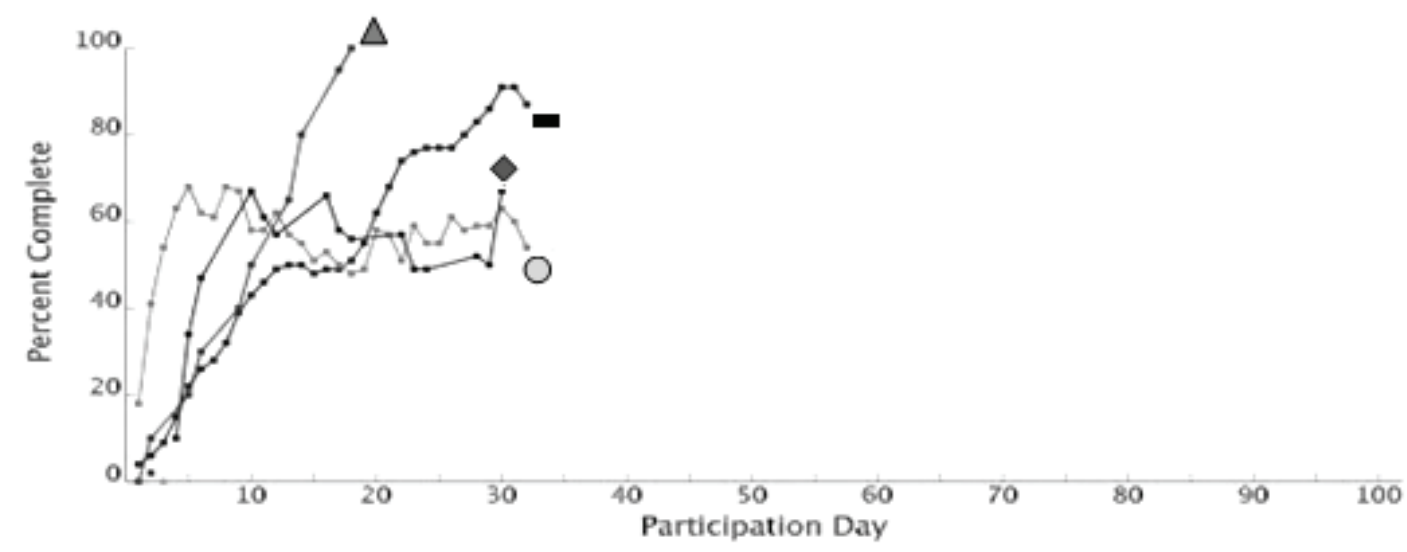

- Circus Sequence (91\%)

Phoneme Identification ( $68 \%$ )

\section{Old MacDonald's Flying Farm (67\%)}

$\triangle$ Phonic Match $(100 \%)$

Fonte: Progress Tracker, Scientific Learning, 2006.

Figura 2 - Desempenho ao longo da aplicação do software FFW do sujeito 2

Sujeito 3 (S3)

As atividades realizadas por S3 foram: circus sequence, phonic match, phoneme identification e Old Mac Donald's Flying Farm. De todas as que S3 demonstrou mais facilidade era a do Old Mac Donald's Flying Farm, porém no circus sequence não demonstrou evolução alguma do primeiro ao último dia de estimulação evidenciando que a tarefa auditiva solicitada foi difícil para S3.

Em relação à escrita de S3 na pré-estimulação foram constatadas alterações importantes evidenciando pouca noção do que é frase ou texto, solici- tação de auxílio e de tempo para pensar em como escrever, não compreensão da variação entre o modo de falar e de escrever, demonstrando não conhecer o sistema ortográfico da língua portuguesa. Apresentou grafia irregular e não fez uso de espaço entre as palavras. $\mathrm{Na}$ análise da escrita de S3 observou-se falta de clareza e coesão. A leitura de S3 foi lenta e silabada, não havendo respeito à pontuação, tentou fazer deduções do que lia e não apresentou adequada compreensão. Na avaliação da leitura e escrita pós-estimulação evidenciouse evolução na organização espacial durante a 
escrita, pois as demais alterações mantiveram-se presentes.

Nas habilidades de consciência fonológica antes da estimulação S3 apresentou $67,5 \%$ de acertos no nível silábico e 50\% no nível fonêmico. Na avaliação após a estimulação observou-se piora do desempenho no nível silábico com $57,5 \%$ de acertos e melhora para $60 \%$ de acertos no nível fonêmico.

Observa-se na Figura 3 o desempenho de S3 ao longo dos dias de intervenção.

Fast Forword Language Completion History

100-Minute Day 1 Protocol: Participation Day 26

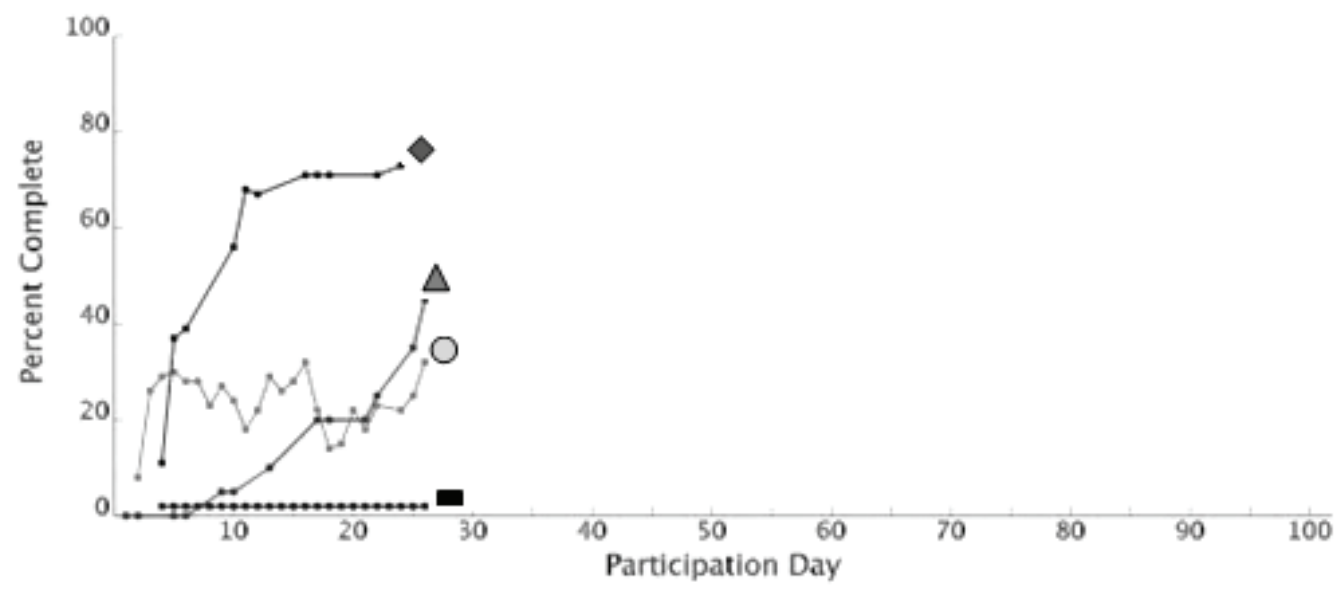

- Circus Sequence $(2 \%)$

Phoneme Identification ( $32 \%$ ) old MacDonald's Flying Farm (73\%)

$\triangle$ Phonic Match $(45 \%)$

Fonte: Progress Tracker, Scientific Learning, 2006.

Figura 3 - Desempenho ao longo da aplicação do software FFW do sujeito 3

\section{Descrição da avaliação comportamental do processamento auditivo antes e após a intervenção}

Serão descritos os resultados da avaliação comportamental do processamento auditivo na seguinte ordem: teste de fala filtrada, teste dicótico de dígitos, teste dissílabos alternados (SSW), teste de padrões de freqüência, teste de padrões de duração e teste de detecção de intervalos de silêncio.

Em relação ao teste de fala filtrada (Tabelas 1 e 2) observou-se que antes da intervenção o S2 apresentou resultado rebaixado, sendo que houve melhora no desempenho deste após a estimulação. Os demais apresentaram resultados dentro da normalidade na pré-estimulação que se manteve normal após a estimulação.

No teste dicótico de dígitos durante a tarefa de integração binaural constatou-se que S1 e S2 apresentaram resultados normais bilateralmente $\mathrm{e}$ S3 apresentou desempenho rebaixado na orelha direita antes da intervenção havendo melhora neste desempenho após a intervenção. Destaca-se, porém que S3 não conseguiu atingir o percentual esperado para a sua idade após a estimulação (Tabela 3).

$\mathrm{Na}$ tarefa de separação binaural pode ser observado na Tabela 4 que o S1 e S2 demonstraram melhora unilateral. Porém, o S3 evidenciou piora após a intervenção.

No teste de dissílabos alternados observou-se maior número de erros nas situações competitivas de escuta, sendo que se evidenciou melhora em todos os sujeitos (Tabela 5).

A respeito do teste padrão de freqüência utilizando a resposta murmurada observa-se que $\mathrm{S} 1$ apresentou desempenho dentro da normalidade bilateralmente, enquanto $\mathrm{S} 2$ foi abaixo do esperado para a idade antes da intervenção normalizando este desempenho após a estimulação. O S3 não conseguiu realizar a tarefa antes e após a estimulação com FFW (Figura 4).

Quanto ao teste padrão de freqüência com resposta verbal observou-se que o S1 apresentou melhora no desempenho em ambas as orelhas 
Tabela 1 - Perceptual de acertos no índice percentual de reconhecimento de fala pré e após a intervenção

\begin{tabular}{rcccc}
\hline & OD - Pré & OD - Após & OE - Pré & OE - Após \\
\hline Sujeito 1 & $92 \%$ & $94 \%$ & $88 \%$ & $90 \%$ \\
Sujeito 2 & $92 \%$ & $100 \%$ & $100 \%$ & $100 \%$ \\
Sujeito 3 & $96 \%$ & $92 \%$ & $84 \%$ & $92 \%$ \\
\hline
\end{tabular}

OD - orelha direita; OE - orelha esquerda

Tabela 2 - Percentual de acerto por orelha no teste de fala filtrada de cada sujeito pré e após a intervenção

\begin{tabular}{rcccc}
\hline & OD - Pré & OD - Após & OE - Pré & OE - Após \\
\hline Sujeito 1 & $68 \%$ & $64 \%$ & $60 \%$ & $72 \%$ \\
Sujeito 2 & $40 \%$ & $72 \%$ & $44 \%$ & $80 \%$ \\
Sujeito 3 & $80 \%$ & $76 \%$ & $80 \%$ & $72 \%$ \\
\hline
\end{tabular}

OD - orelha direita; OE - orelha esquerda

Tabela 3 - Percentual de acertos no teste dicótico de dígitos na tarefa de integação binaural antes e após a intervenção

\begin{tabular}{lcccc}
\hline & OD - pré & OD - após & OE - pré & OE - após \\
\hline Sujeito 1 & $90 \%$ & $95 \%$ & $82,50 \%$ & $95 \%$ \\
Sujeito 2 & $95 \%$ & $100 \%$ & $92,5 \%$ & $97,50 \%$ \\
Sujeito 3 & $60 \%$ & $87,50 \%$ & $95 \%$ & $75 \%$ \\
\hline
\end{tabular}

OD - orelha direita; OE - orelha esquerda

Tabela 4 - Percentual de acertos no teste dicótico de dígitos na tarefa de separação binaural antes e após a intervenção

\begin{tabular}{ccccc}
\hline & $\begin{array}{c}\text { atenção } \\
\text { a direita - pré }\end{array}$ & $\begin{array}{c}\text { atenção } \\
\text { a direita - após }\end{array}$ & $\begin{array}{c}\text { atenção } \\
\text { a esquerda - pré }\end{array}$ & $\begin{array}{c}\text { atenção } \\
\text { a esquerda - após }\end{array}$ \\
\hline Sujeito 1 & $72,50 \%$ & $75 \%$ & $47,50 \%$ & $72 \%$ \\
Sujeito 2 & $85 \%$ & $95 \%$ & $92,50 \%$ & $92,50 \%$ \\
Sujeito 3 & $87,50 \%$ & $52,50 \%$ & $80 \%$ & $50 \%$ \\
\hline
\end{tabular}

Tabela 5 - Número de erros nos totais combinados do teste dissílabos alternados pré e após a intervenção

\begin{tabular}{ccccccccc}
\hline & $\begin{array}{c}\text { DNC - } \\
\text { Pré }\end{array}$ & $\begin{array}{c}\text { DNC- } \\
\text { Após }\end{array}$ & $\begin{array}{c}\text { DC- } \\
\text { Pré }\end{array}$ & $\begin{array}{c}\text { DC - } \\
\text { Após }\end{array}$ & $\begin{array}{c}\text { EC - } \\
\text { Pré }\end{array}$ & $\begin{array}{c}\text { EC - } \\
\text { Após }\end{array}$ & $\begin{array}{c}\text { ENC - } \\
\text { Pré }\end{array}$ & $\begin{array}{c}\text { ENC - } \\
\text { Após }\end{array}$ \\
\hline Sujeito 1 & 5 & 3 & 10 & 10 & 15 & 5 & 3 & 1 \\
Sujeito 2 & 1 & 0 & 7 & 3 & 5 & 0 & 0 & 0 \\
Sujeito 3 & 4 & 1 & 7 & 5 & 15 & 10 & 8 & 2 \\
\hline
\end{tabular}

DNC - Direita não competitiva; DC - Direita competitiva; EC - Esquerda competitiva; ENC - Esquerda não competitiva

após a intervenção atingindo o padrão de normalidade. O S2 apresentou um melhor desempenho somente da orelha esquerda após a estimulação e S3 não conseguiu realizar o teste antes e também após a estimulação do FFW (Figura 5).

A respeito do teste padrão de duração com res- posta verbal observa-se que o S1 e S2 apresentaram melhores desempenhos de ambas as orelhas após a estimulação com o FFW e o sujeito 3 não conseguiu realizar a tarefa antes da estimulação e após a estimulação o sujeito conseguiu realizar, contudo não atingindo a normalidade (Figura 6). 


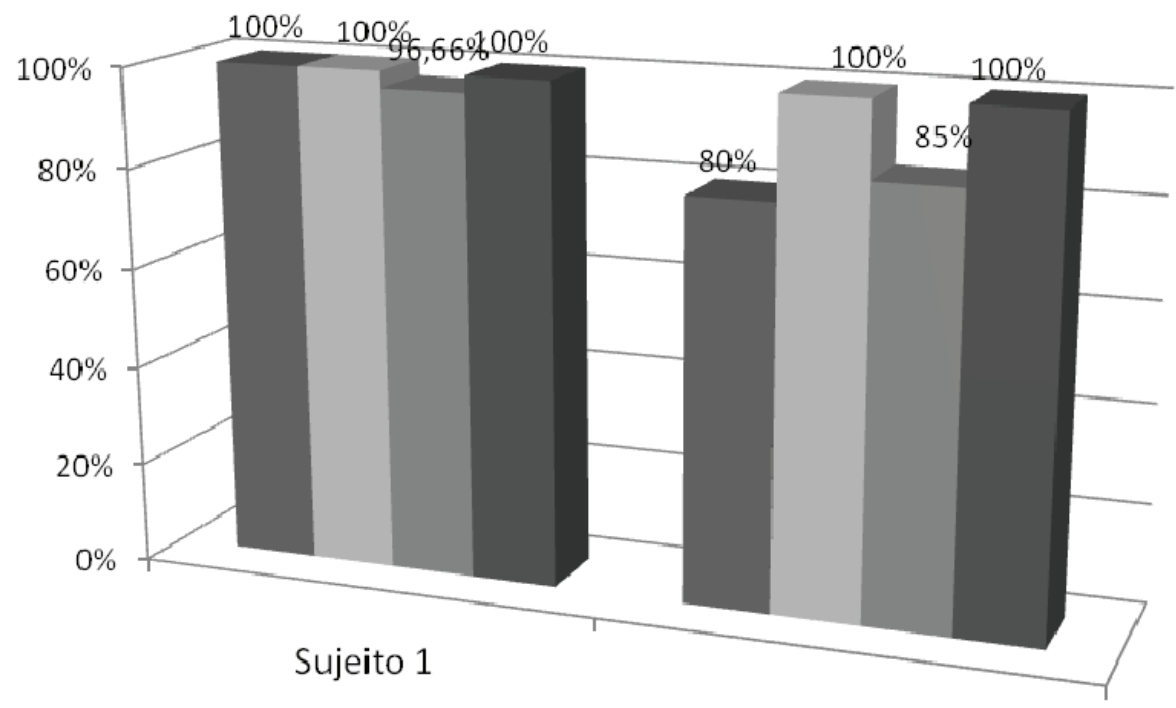

Sujeito 2

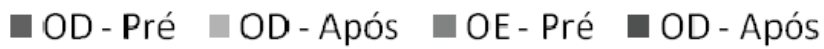

Figura 4 - Percentual de acertos por orelha no teste de padrões de freqüência utilizando a resposta de murmúrio pré e após a intervenção do S1 e S2

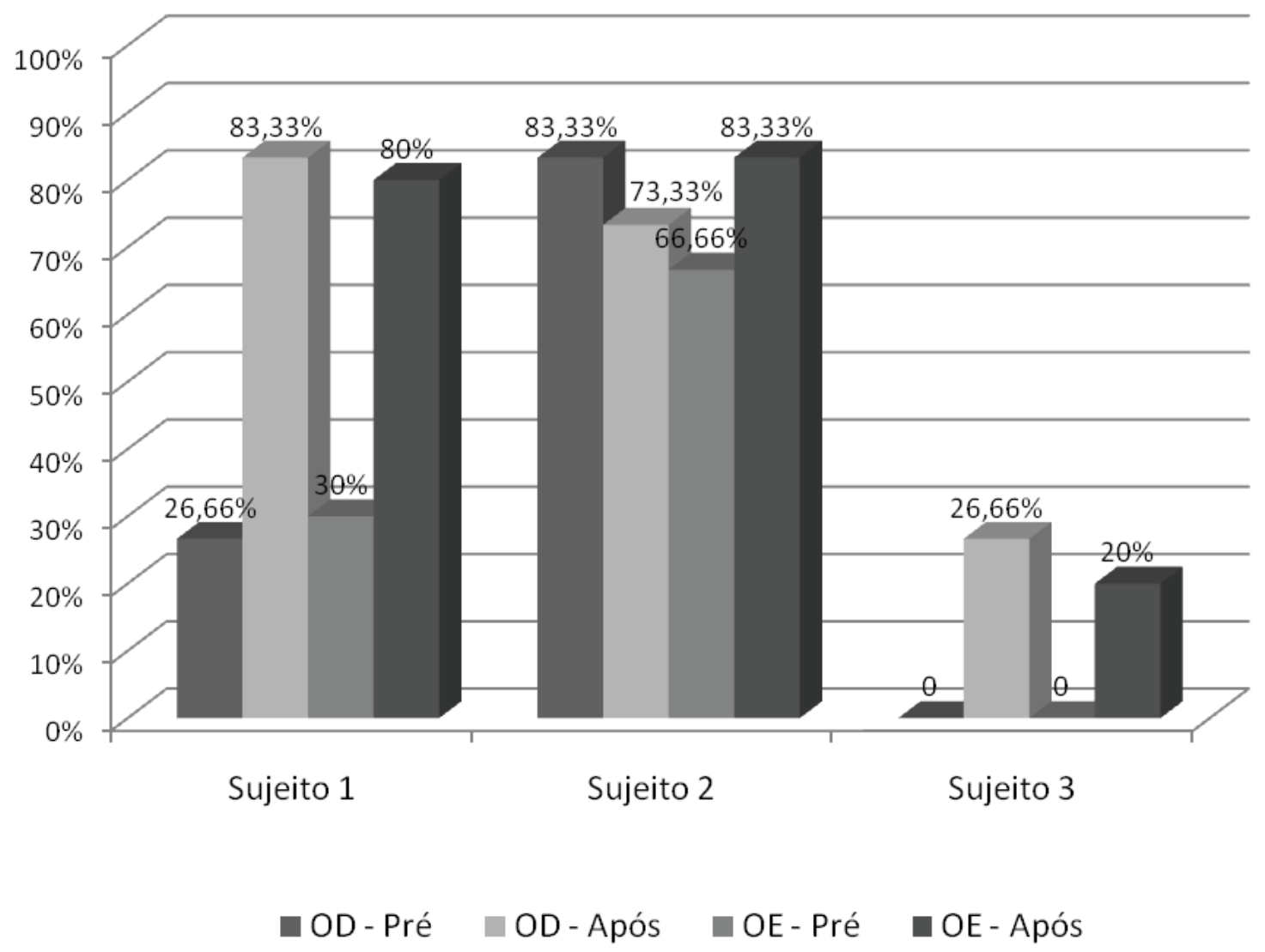

Figura 5 - Percentual de acertos por orelha no teste de padrões de freqüência utilizando a resposta verbal pré e após a intervenção de S1, S2 e S3 


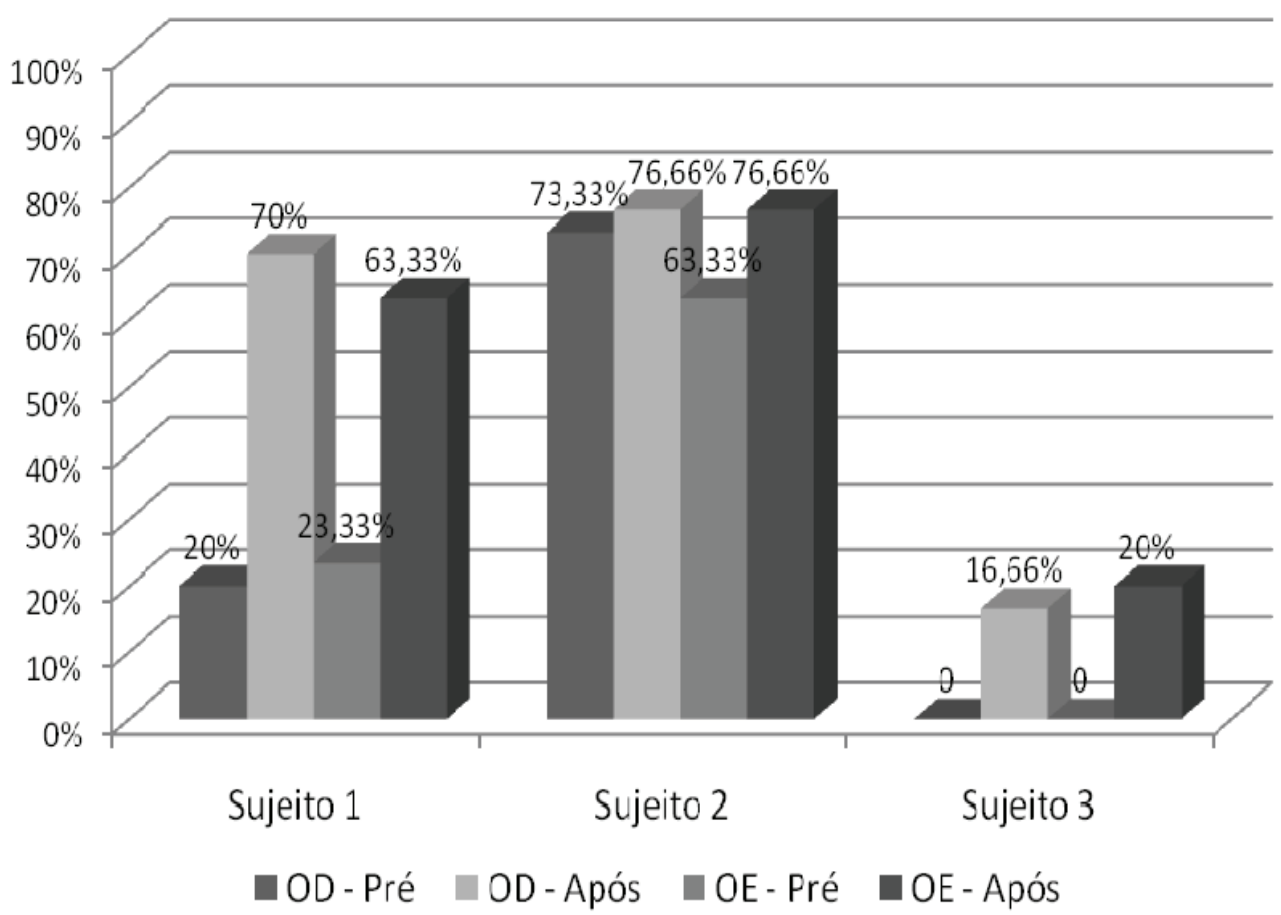

Figura 6 - Percentual de acertos por orelha no teste de padrões de duração utilizando a resposta verbal pré e após a intervenção de S1, S2 e S3

Tabela 6 - Média do limiar de detecção de intervalos de silêncio para as freqüências de 500 a $4000 \mathrm{~Hz}$ no teste RGDT antes e após a intervenção

\begin{tabular}{ccc}
\hline & Pré & Após \\
\hline Sujeito 1 & Não reconheceu $300 \mathrm{~ms}$ & $50 \mathrm{~ms}$ \\
Sujeito 2 & $8,5 \mathrm{~ms}$ & $2,25 \mathrm{~ms}$ \\
Sujeito 3 & Não reconheceu $300 \mathrm{~ms}$ & Não reconheceu $300 \mathrm{~ms}$ \\
\hline
\end{tabular}

*Média obtida de 1000, 2000 e $4000 \mathrm{~Hz}$.

O teste RGDT revelou que o S1 e S2 melhoraram seu desempenho após a estimulação e o S3 ficou estagnado, não reconheceu 300 milisegundos antes e nem após a aplicação do software (Tabela 6).

\section{DISCUSSÃO}

Primeiramente, é importante discutir a presença do distúrbio do processamento auditivo associado aos transtornos de leitura e escrita dos sujeitos investigados, bem como o tipo de distúrbio de processamento auditivo presente e, posteriormente, discutir os resultados encontrados antes e após a intervenção com o software Fast ForWord.

A relação entre os distúrbios do processamento auditivo (central) e os transtornos de leitura e escrita/ dislexia é descrita em diferentes pesquisas ao longo das últimas décadas ${ }^{2,3,17,18}$.
Os autores do software Fast ForWord em suas pesquisas básicas, isto é, anteriores e de fundamentação para a criação do FFW apontaram que os distúrbios específicos de linguagem são causados por problemas no processamento temporal da informação auditiva ${ }^{7,8,10}$. Mais tardiamente outras pesquisas descreveram resultados de ressonância magnética funcional antes e após o uso do software Fast ForWord em adultos e crianças disléxicas, evidenciando modificações em diferentes regiões cerebrais, principalmente, na região pré-frontal, temporo-parietal e giro hipocampal esquerdo após o uso do FFW ${ }^{17,18}$.

O software Fast ForWord propõe-se a desenvolver essencialmente o processamento temporal, buscando que o processamento auditivo torne-se mais eficiente e eficaz para auxiliar, conseqüentemente, no desenvolvimento de linguagem e de leitura e escrita. $O$ treinamento especializado de tarefas 
lingüísticas e perceptuais prove uma via principal para realçar as habilidades comunicativas receptivas e expressivas e isso foi evidenciado pelo uso do FFW em crianças com implante coclear ${ }^{15}$.

Diante dos resultados obtidos antes da aplicação do software Fast ForWord e na comparação desses com os resultados após aplicação em três crianças brasileiras, pode-se constatar algumas mudanças nos sujeitos da presente pesquisa evidenciando que o FFW possa ter atingido seus objetivos no que se refere ao processamento auditivo, em especial, habilidades do processamento temporal, pois observou-se melhora nas habilidades de ordem e seqüência temporal. Este aspecto não foi observado em duas pesquisas utilizando o teste de mascaramento temporal antes e depois da intervenção com o FFW em crianças com distúrbios de linguagem e de aprendizagem ${ }^{8,10}$. Isto evidencia que é possível que o FFW auxilie no desenvolvimento de algumas habilidades do processamento temporal e não na totalidade destas habilidades temporais, destacando-se que o reconhecimento de padrões temporais foi a habilidade que mais se modificou após a intervenção com o FFW, principalmente, da resposta verbal no teste de padrões de freqüência e de duração.

O tipo de distúrbio de processamento auditivo (central) apresentado pelos sujeitos desta pesquisa na pré-estimulação era predominantemente do tipo associação auditivo-lingüístico em S1, de decodificação e associação auditivo-lingüístico em S2 e de integração auditiva e/ou prosódia no S3 ${ }^{19}$.

Após a intervenção observou-se que S1 e S2 atingiram normalidade no processamento auditivo e S3 embora apresentasse evoluções permanecia com dificuldades de integração auditiva evidenciado pelo número de erros no teste SSW na orelha esquerda competitiva, bem como pela dificuldade nos testes de padrões de freqüência e de duração com resposta verbal. Entretanto, há evidências pelo desempenho no teste de padrões de freqüência com resposta murmurada de dificuldades de prosódia. Com base nestas colocações há de se observar que talvez o software FFW proporcione poucas atividades específicas para desenvolvimento do déficit de prosódia e de integração auditiva ou o tempo de intervenção realizada por S3 tenha sido insuficiente para que o mesmo tivesse evoluções satisfatórias.

A estratégia que S3 apresentou menos evoluções foi o Circus Sequence que exigia dele reconhecimento e discriminação do pitch com variação na duração e intervalo inter-estímulo. Destaca-se que o reconhecimento do pitch exigido nesta estratégia, assim como a tarefa murmurada do teste de padrões de freqüência que S3 não conseguiu desempenhar pré e pós-estimulação são realizadas pelo hemisfério direito, havendo necessidade de integração inter-hemisférica para realização da resposta verbal ${ }^{20}$.

Os demais sujeitos evidenciaram evoluções no Circus Sequence apontando para que as dificuldades com esta estratégia estivessem relacionadas especificadamente com S3.

Constatou-se que houve melhora na atenção sustentada de todos os participantes, porque as reavaliações do processamento auditivo puderam ser realizadas em uma única sessão, enquanto as avaliações pré-estimulação foram realizadas em três sessões, em virtude do cansaço e queda da atenção ao longo destas. Esta observação qualitativa dos sujeitos concorda com a indicação de que testes padronizados nem sempre são a melhor opção para verificar mudanças antes e após a aplicação do software FFW, sendo necessário a inclusão de observação da comunicação em contexto natural, observação da criança no ambiente e aplicação de questionário com pais e professores ${ }^{8}$.

Desta forma, há evidências quantitativas e qualitativas de mudanças no processamento auditivo após o uso do FFW e isso evidencia, conseqüentemente, plasticidade do sistema nervoso central propiciado pela estimulação auditiva diária e intensiva realizada pelo software ${ }^{18}$.

Diante deste fato, pode-se perceber a importância do treinamento auditivo no tratamento de crianças com distúrbio do processamento auditivo relacionado à sua intensidade e freqüência.

Em relação à freqüência do treinamento auditivo é importante destacar as dificuldades encontradas, nesta pesquisa, em conseguir que as famílias e as crianças se dispusessem a realizar o programa 80 minutos por dia, cinco dias por semana ao longo de até oito semanas. Assim, devem-se buscar formas alternativas para a aplicação do FFW que sejam eficazes, tais como o estudo em que verificou a viabilidade dos pais administrarem a utilização do FFW por seus filhos monitorados por um profissional $\mathrm{e}$ constataram que $75 \%$ das crianças apresentaram benefícios ${ }^{9}$.

O uso de um software como o Fast ForWord proporciona registro fidedigno do tempo de resposta, número de tentativas, acertos e erros realizados pela criança que se mostra uma ferramenta importante para o fonoaudiólogo monitorar o processo terapêutico com paciente, tendo em vista que apresenta o desempenho diário em gráficos que são úteis tanto para o planejamento terapêutico quanto para explanar os resultados para os familiares e outros profissionais ${ }^{7}$. Soma-se a este aspecto que o software é utilizado independente do processo de avaliação e diagnóstico dos sujeitos, fornecendo 
assim liberdade ao terapeuta para medir o sucesso do programa utilizando medidas padronizadas antes e após o seu uso ${ }^{7}$.

Outro aspecto positivo evidenciado pela experiência em aplicar o FFW foi o controle sobre o sinal acústico e visual utilizado na intervenção que não oferecem outras pistas supra-segmentais ou sociais que são inerentes na relação inter-pessoal da terapia tradicional e que em alguns tipos de distúrbios do processamento auditivo (central) devem ser minimizados para que as estratégias sejam desafiadoras e auxiliem a realizar mudanças no sistema nervoso auditivo central ${ }^{6}$. Da mesma forma, o controle sobre os aspectos acústicos do som, principalmente, no que se refere à graduação da duração e a intensidade são possíveis apenas por estimulações controladas ${ }^{6}$ como as utilizadas pelo FFW.

$\mathrm{Na}$ atualidade, as crianças são estimuladas muito precocemente ao uso de computadores e isso se torna um atrativo quando utilizado em terapia fonoaudiológica. Além do que os softwares também utilizam designs que auxiliam na intensidade da intervenção, pois apresentam animações e gráficos visuais que auxiliam na atenção e encorajam a criança a interagir com o software mais tempo do que algumas terapias tradicionais ${ }^{6,7}$.

Um aspecto importante observado com o uso do FFW no Brasil foi a pouca autonomia que o software proprocionou aos pesquisadores na escoIha das estratégias que serão liberadas para uso a cada dia. Como o software Fast ForWord Language é composto por sete estratégias, sendo que três delas exigem conhecimento da língua inglesa não puderam ser aplicadas com a amostra desta pesquisa. Desta forma, a cada dia no protocolo de 100 minutos eram liberadas pelo sistema do FFW cinco estratégias que deveriam ser jogadas 20 minutos cada. Estas estratégias não podiam ser escolhidas pelos pesquisadores, sendo liberadas igualmente pelo sistema para todas as crianças. Por este motivo que uma estratégia era realizada novamente na versão demonstração para completar a estimulação de 80 minutos.

Ao analisar a avaliação de linguagem escrita antes e após a estimulação constatou-se que não existiram muitas modificações, sendo estas observadas no processamento auditivo central e na consciência fonológica; porém se acredita que este aspecto era o esperado tendo em vista que as três estratégias do Fast ForWord Language que envolvem linguagem não foram utilizadas na estimulação das crianças Brasileiras, porque envolviam domínio da língua inglesa.

Destaca-se que os dados apresentados nesta pesquisa são iniciais, havendo necessidade de ampliar a amostra de crianças brasileiras com a aplicação do software FFW para obter conclusões mais precisas e generalizantes. Por outro lado o software FFW apresenta-se como interessante instrumento e exemplo de programa terapêutico.

No Brasil deve-se investir na criação de novos instrumentos para a língua Portuguesa tendo como referência os princípios, os fundamentos e resultados de aplicabilidade deste software em vários locais do mundo, bem como da alta competência científica de seus fundadores.

\section{CONCLUSÃO}

Com base na experiência descrita de aplicação do software Fast ForWord em três crianças brasileira com distúrbios do processamento auditivo pode-se concluir que é possível ter benefícios com a aplicação deste software no Brasil. No entanto, dois fatores podem ser empecilhos para o uso efetivo do software Fast ForWord em novas pesquisas e no contexto clínico no Brasil. Estes fatores referem-se à estimulação cinco vezes por semana que traz dificuldades importantes para as famílias aderirem ao tratamento e o alto custo comercial deste programa.

Neste contexto, novas pesquisas com ampliação da amostra de sujeitos Brasileiros expostos a este software podem auxiliar a determinar a eficácia de sua aplicação e dar subsídios que fundamentem o investimento e o tempo de sua aplicação por fonoaudiólogos no Brasil.

\section{AGRADECIMENTOS}

Os autores agradecem a colaboração durante a pesquisa de campo dos alunos do Curso de Fonoaudiologia da UNIVALI Ademir Comerlatto Junior, Graziela Liebel, Letícia Bretzke, Mariane Perin da Silva, Miriam Regina Boeno e da Fonoaudióloga Carla Meller Mottecy. 


\begin{abstract}
Background: news proposals of rehabilitation for children with auditory processing disorders associated to language and learning disorders are necessary to increase the efficacy of speech and language treatment. So, the purpose was to check the applicability of Fast Forword (FFW) software for managing auditory processing disorder (APD) in three Brazilian kids. Procedure: these three children, ranging from 9 to 14-year old, were selected in basic auditory evaluation, auditory process evaluation, language evaluation and phonological awareness. These tests applied before and after the training with FFW, ran during 100 minutes a day, five days a week along eight weeks. The following strategies were applied: Circus sequence, Old Mac Donald's Flying Farm, Phoneme identification and Phonic Match, involving detection, discrimination, sustained attention and auditory memory. Results: after about 30.67 days using FFW, we noted adequacy in auditory process of two kids. In one of the kids that were not the case, the same alterations showed in the auditory process re-evaluation. After a third week of stimulation a diminution of interest for the strategies was observed, which demanded a more intense and creative intervention of the researchers. Conclusion: it is possible to infer that FFW presents applicability for Brazilian kids with auditory process disturbances; however, research with larger samples is necessary in order to check the effectiveness of this software on Brazilian kids.
\end{abstract}

KEYWORDS: Hearing; Child; Auditory Perception; Acoustic Stimulation

\section{REFERÊNCIAS}

1. Muniz LF, Roazzi A, Schochat E, Teixeira CF, Lucena JA. Avaliação da habilidade de resolução temporal, com uso do tom puro, em crianças com e sem desvio fonológico. Rev CEFAC. 2008; 9(4):550-62.

2. Neves IF, Schochat E. Maturação do processamento auditivo em crianças com e sem dificuldades escolares. Pró-Fono. 2005; 17(3):311-20.

3. Sauer L, Pereira LD, Ciasca SM, Pestun M, Guerreiro MM. Processamento auditivo e SPECT em crianças com dislexia. Arq Neuropsiquiatr. 2006; 64(1):108-11.

4. American Speech-language-hearing association (ASHA). (Central) Auditory Processing Disorders. 2005. Disponível em: <http://www.asha.org/ members/deskref-journals/deskref/default>. Acesso em: 25/03/2006.

5. Musiek FE, Chermak GD, Weihing J. Auditory training. In: Chermak GD, Musiek FE. Handbook of (central) auditory processing disorder: comprehensive intervention. San Diego: Plural Publishing; 2007. p. 77-106.

6. Thibodeau LM. Computer-based auditory training (CBAT) for (Central) auditory processing disorders. In: Chermak GD, Musiek FE. Handbook of (central) auditory processing disorder: comprehensive intervention. San Diego: Plural Publishing; 2007.

7. Veale TK. Targeting temporal processing deficits through Fast Forword: language therapy with a new twist. Lang Speech Hear Serv Schools. 1999; 30:353-62.
8. Gillam RB. Computer-assisted language intervention using Fast Forword: theorical and empirical considerations for clinical decision-making. Lang Speech Hear Serv Schools. 1999; 30:363-70.

9. Loeb DF, Stoke C, Fey ME. Language changes associated with Fast ForWord-language: evidence from case studies. Am J Speech Pathol. 2001; 10(3):216-30.

10. Marler JA, Champlin CA, Gillam RB. Backward and simultaneous masking measured in children with language-learning impairments who received intervention with Fast ForWord or Laureate Learning Systems Software. Am J Speech Lang Pathol. 2001; 10:258-68.

11. Troia GA, Whitney SD. A close look at the efficacy of Fast ForWord Language for children with academic weaknesses. Contemp Educ Psychol. 2003; 28:465-94.

12. Rouse CE, Krueger AB. Putting computerized instruction to the test: a randomized evaluation of a "scientifically based" reading program. Econ Educ Rev. 2004; 23(4):323-38.

13. Cohen W, Hodson A, O'Hare A, Bayle J, Durrani T, McCartney E et al. Effects of computerbased intervention through acoustically modified speech (Fast ForWord) in severe mixed receptiveexpressive language impairment: outcomes from a randomized controlled trial. J Speech Lang Hear Res. 2005; 48:715-29.

14. Valentine D, Hedrick MS, Swanson LA. Effect of an auditory training program on reading, phoneme awareness, and language. Percept Motor Skills. 2006; 103(1):183-96. 
15. Schopmeyer B, Mellon N, Dobaj H, Grant G, Niparko JK. Use of Fast ForWord to enhance language development in children with cochlear implants. Ann Otol Rhinol Laryngol Suppl. 2000; 185:95-8.

16. Gillam RB, Loeb DF, Hoffman LM, Bohman T, Champlin CA, Thibodeau $L$ et al. The efficacy of Fast ForWord language intervention in school-age children with language impairment: a randomized controlled Trial. J Speech Lang Hear Res. 2008; 51:97-119.

17. Temple E, Poldrack RA, Protopapas A, Nagarajan S, Salz T, Tallal P et al. Disruption of the neural response to rapid acoustic stimuli in dyslexia: evidence from functional MRI. Proc Natl Acad Sci U S A. 2000; 97(25):13907-12.

18. Temple E, Deutsch GK, Poldrack RA, Miller SL, Tallal P, Merzenich MM, Gabrieli JDE. Neural deficits in children with dyslexia ameliorated by behavioral remediation: evidence from functional MRI. Proc Natl Acad Sci USA. 2003; 100(5):2860-5.

19. Bellis TJ. When the brain can't hear. New York: Atria Books; 2002.

20. Musiek FE, Pinheiro M, Wilson DH. Auditory pattern perception in "split brain" patients. Arch Otolaryngol. 1980; 106(10):610-2.

Endereço para correspondência:

Rua Rui Barbosa, 372 ap. 2

Balneário Camboriú - SC

CEP: 88331-510

Tel: (47) 3367-0949 / (48) 9980-4687

E-mail: sheilabalen@ univali.br /

sheila@sheilabalen.com.br 